\title{
LA-UR $94-3199$
}

TITLE: "Oxidation Behavior of Aluminum Nanopowders"

$\begin{array}{lll}\text { AUTHOR(S): } & \text { Christopher E. Aumann } & \text { CST-10 } \\ \text { Gretchen L. Skofronick } & \text { CST-10 } \\ & \text { Joe A. Martin } & \text { CST-10 }\end{array}$

SUBMITTED To: Journal of Vacuum Sclence and Technology Proceedings of the 4 lst National Symposium of the American Vacuum Society

\section{DISCLAIMER}

This report was prepared as an account of work sponsored by an agency of the United Stales Government. Neither the Unite' States Government nor any agency thereor, nur any of their employees, makes uny warranty, express or implied, or assumes any legal liability or responsibility for the accuracy, compieleness, or usefulness of any information, apparalus, prodict, or procest disclosed, or represents that its use would not infringe privalely owned rights. Reference herein to any specific commercial product, process, or service by trade name, Irademark. manufaclurer, ur clherwise does not ne essarily constitute or imply its endorsement, recommendation, or favoring by the Uniled Stales Government or any agency thereof. The views and opinions of authors expressed herein do not necessarily state or reflect these of the Uniled Stales Goverrment or any agency thereof. 


\title{
Oxidation Behavior of Aluminum Nanopowders \\ C.E. Aumann, G.L. Skofronick, and J.A. Martin Chemical Science and Technology Division Los Alamos National Laboratory Mail Stop K765, Los Alamos, NM 87545
}

\begin{abstract}
Alestract
Rutherford backsuattering spectrometry, thermogravimetric analysis, and high-resolution transmission electron rnicroscopy were used to investigate the oxidation behavior of ultrafine grain aluminum powder. Fractional change in mass of Al powder samples were obtained as a function of temperature and exposure time for samples with different particle size distributions. Activation energy for oxidation is found to depend on average $\mathrm{Al}$ particle size and to be much smaller than that known for flat $\mathrm{Al}$ samples.
\end{abstract}

\section{Introduction}

Interest in nanostructures is driven by the potential for finding novel prcperties ir. material structures that have small physical dimensions. Composites constructed from nanosized components also possess useful properties that depend on the size and characteristics of the individual constituents. An example is found in ultrafine grain (UFG) powder mixtures of thermite-like materials which exhibit substantially greater reaction rates than thermite ( $\mathrm{Al}$ and $\mathrm{Fe}_{2} \mathrm{O}_{3}$ ) mixtures. Specifically, $\mathrm{Al}$ and $\mathrm{MoO}_{3}$ powder samples with average particle size of 0.02 $0.05 \mu \mathrm{m}$ react more than 1000 imes faster than conventional powdened thermite owing to reduced diffusion distances between individual reactant species. Reaction enthalpy of a stoichiometric mixture of $\mathrm{Al}$ and $\mathrm{MoO}_{3}$ is $-1.12 \mathrm{kcal} / \mathrm{gm}$, which is $12 \%$ greater than that of TNT. Energetic metastab e mixtures of these two highly reactive substances are possible because each $\mathrm{Al}$ particle consists of an aluminum core surrounded by a shell of amorphous alumina. Core $\mathrm{Al}$ remains isolated from oxidizer material until external energy dumped into the composite causes oxide sheils to fail resulting in initiation of a self-propagating reaction. Reactive power, i.e., the 
rate at which energy is released, depends on a number of factors including particle size distribution, degree of intermixing of $\mathrm{Al}$ and oxidizer powders, mean oxide thickness, and physical characteristics of the amorphous alumina shells. Total energy released from a metastable energetic composite ii a function of reaction stoichiometry, i.e., the relative amount of pure Al to oxidizer, which is a function of oxide thickness and size distribution of UFG Al powder. Because reactive power depends on several factors that can be manipulated during the powder growth process, energetic metastable composite materials can be designed for applications that require a specific energy release rate. Other propertics of UFG powder reactions such as ignition threshold energy also depend on physical characteristics of UFG Al powder. Amorphous alumina shells in as-prepared UFG Al are approximately $30 \AA$ thick. As a cunsequence, $\mathrm{UF} / \mathrm{Al} / \mathrm{MoO}_{3}$ mixtures are highly sensitive to ignition by electrical spark. The oxidation study reported here was in part motivated by the need to find a means to desensitize UFG composites to ignition by electrical spark or friction. The premise is that ignition threshold energy could be raised sufficiently by increasing oxide shell thickness to yield a composite material that is safe to handle in large quantities. Previous aluminum oxidation studies $(1-3)$ have been performed investigating mass changes that occur during oxide film formation on nominally flat surfaces. An oxidation study of nanoparticles provides an opportunity to explore effects of surface energy on fundamental processes such as diffusion because surface energy contributes significantly to total energy for small paricle sizes. Often, properties of nanosized components can be inferred from macroscopic changes that occur in a bulk quantity of material. In this study, thermogravimetric analysis (TGA) and Rutherford backscattering spectrometry (RBS) have been used to measure changes in UFG Al powder samples annealed at fixed teinperatures during exposure to oxygen. Activation energy was determined from a series of isothermal mass change measurements taken at different temperatures for powder samples with different particle size distributions. Powder samples were initially characterized using high-resolution transmission electron microscopy (HRTEM). 


\section{Experimental Procedure}

\section{A. Powder Synthesis}

Ultrafine grain Al powders are synthesized using dynamic gas condensation, a technique developed by several researchers. ${ }^{(4)}$ The techrique consists of lieating a metal to produce a flux of metal atoms which interact with a low pressure inert background gas (typically Argon at 1-10 torr). Metal atoms entering into vapor phase are slowed sufficiently by collisions with inert gas atoms to enable nucleation and growth of small metal particles. Resistive or if induction heating methods are used to heat $\mathrm{Al}$ to about $300^{\circ} \mathrm{C}$, a temperature where vapor pressure flux of $\mathrm{Al}$ is sufficient to yield efficient nucleation and growth. UFG $\mathrm{MoO}_{3}$ powder is fabricated similarly by heating molybdenum meial in an oxygen background. UFG particles are carried to cooler surfaces by convective gas currents 'where they agglomerate and are later retrieved as powder. Mean particle size is varied by adjusting me'al source temperature and background gas pressure. In the case of UFG Al, powder retrieval is performed following a post-growth oxidation procedure which consists of a controlled admittance of air into the growin chamber after powder samples reach room temperature. The procedure results in the formation of a thin oxide shell of alumina around eacin $\mathrm{Al}$ particle which prevents UFG Al from being pyrophonc. Retrieved UFG powder is passed through a 120 mesh sieve to break up large agglomerates. Fine-scale mixing is achieved by ultrasonication of powders in a liquid suspension agent such as isopropanol.

\section{B. Pow Jer Characterization}

As-prepared Al powder is characterized with high-resolution transmission electron microscopy to identify structure and size distribution of UFG Al particles and with Rutherford backscattering spectrometry to determine average oxygen content. Figure 1 is an HRTEM micrograph of an UFG Al particle which reveals that particle structure consists of a crystalline aluminum core surrounded by an amorphous alumina shell. Figure 2 is an HRTEM micrograph illustrating that UFG Al particles exhibit a distribution of sizes and can be fabricated with average diameters of about $0.02 \mu \mathrm{n}$. Growth conditions are varied to obtain particle ensembles 
with different physical characteristics. RBS and YRTEM are complementary because the fraction of oxygen in UFG Al powder samples is a function of both average oxide thickness and particle size distribution. RBS measurements of pressed pellers of UFG Al powder nrovide a large scale average of oxygen content while HRTEM measurements of $\mathrm{Al}$ particles supply size and oxide thickness values of individual particles. Determination of particle size distribution from FRTEM is time intensive and limited to these particles that are smail encugh for transmission by the electron beam. However, HRTEM and RBS together provide size distribution information not generally available using standard sizing techniques which have difficulty probing sizes on the order of $0.05 \mu \mathrm{m}$ or less. In RBS spectra from pressed UFG Al powder, atomic percent oxygen is determined from a ratio of the height of the oxygen peak relative to $\mathrm{Al}$ once peak heights are scaled to accurately known scattering cross sections. In a powder sample of monosized spherical Al particles, atomic fraction of oxygen in the sample is given by

$$
\% O=3\left[5+\gamma \beta\left(x^{3}-1\right)^{-1}\right]^{-1}
$$

where $\gamma=0.68$ is the ratio of $\mathrm{Ai}$ to alumina densities, $\beta=3.78$ is the ratio of alumina to $\mathrm{Al}$ molecular weights, and $\mathrm{x}$ is the ratio of particle to core radii, $\tau_{\mathrm{p}} / \tau_{\mathrm{c}}$. Becpuse the core radius $\mathrm{r}_{\mathrm{c}}=$ $\mathrm{r}_{\mathrm{p}}-\delta(\delta=$ oxide thickness $), \mathrm{x}=1+\delta / \mathrm{r}_{\mathrm{c}}$. From this relation a sample of $400 \AA$ diameter paricles each with a $10 \AA$ thick oxide shell would measure an atomic fraction of oxygen of $15 \%$, a value easily detected with RBS.

Three batches of UFG Al powder were synthesized for this study. RBS measurements of oxygen content in saraples referred to as $\mathrm{A}, \mathrm{B}$, and $\mathrm{C}$ were 32,18 , and 13 atomic percent, respectively. HRTEM measurements of average oxide thickness in as prepared UFG Al were 29 , 38 , and $36 \AA$ for $A, B$, and C, respectively. No correlation between oxide thickness and particle size was observed within a given sample. From equation [1] these oxide thicknesses and atomic $\% O$ values correspond to particle diameters cí 406,1151 , and $1665 \AA$, respectively. However, 
actual particle size distributions are not monosized. As shown in figure 3 for sample A, UFG particle size distributions measured by HRTEM exhibit a probability tail that extends well beyond its peak value. Average particle size of a realistic distribution is much less than that predicted from [1] because atomic \%O decreases with particle size for fixed oxide thickness. UFG Al size distributions have been fit to a log-normal probability function that has been shown to accurately model UFG particle sizes grown via inert-gas condensation(5). Values for average particle diameter, surface area, and atomic $\% \mathrm{O}$ are readily determined from combinations of moments of the log-normal distribution assurning spherical particles and fixed oxide thickness. The un aid curve in figure 3 is a log-normal curve of the form exp $\left(-(\ln (r)-\ln (\Delta))^{2} /\left(2 \ln ^{2} \sigma\right)\right)$ with $\Delta$ chosen to match the peak position determined by HRTEM and $\sigma$ chosen such that the distribution has an atomic $\% O$ value that matches that determined from RBS. Because these powder samples nominally received equivalent up-to-air procedures, oxygen content differences reflect differences in particle size distribution. Samples A, B, and C were fit by log-normal distributions with $(\Delta, \sigma)=(83, \therefore 64),(113,1.96)$, and $(163,1.97)$ angstroms, respectively, which correspond to average particle diameters of 240,445 , and $650 \AA$. It is important to have an accurate determination of particle size distribution because total mass change during oxidation depends on total exposed surfi se area of a given aliquot of sample. Mass uptake values for aliquots from a given sample can be compared directly after they have been scaled to aliquot mass. However, the magnitude of scaled mass uptake values will vary for samples with different surface area to mass ratioj. A monosized distribution of UFG Al powder is of course preferred, but not possible due to the nature of inert-gas condensation.

\section{Oxidation Procedure}

Tw'o systems were used to oxidize UFG Al powder. One was a straightforward gas fiow unit employed to oxidize (IFG Al powder in quanticies sufficient for RBS analysis. It consists of a quartz tube housed within a temperature controlled oven with its ends connected to a gas manifold. $O_{2}$ is passed over loose powder material held in an alumina crucible that has been 
inserted into the quartz tube; flow rate is $0.2 \mathrm{scf:n}$. Oven temperature is then ramped up to a specified temperature and held for a rixed period of time. At the end of a heating cycle samples are removed from the oven and cool rapidly uncier flowing oxygen. Tile second system is a Perkin-Elmer thermogravimetric analysis unit which provides a mass gain or mass uptake history of a sample taken to a specified temperature while exposed to flowing oxygen gas. Temperature ramp up times were approximately 15 minutes for both methods. Samples appeared to be sufticient'y outgassed by this time as no weight loss was observed after the ramp up period. Thermogravimetric measurements are useful for identifying the time dependence of an oxidation process while post-c,xidation RBS and HRTEM measurements are useful to assess changes in particle structure and composition. Results of both types of oxidation experiments are summarized in the following section.

\section{Results anu' Analysis}

Figure 4 is a plot of oxygen content (measured using RBS) in sample A versus annealing temperature for different oxygen exposure times. Sample $A$ had an as-prepared oxygen content of 32 atomic $\% \mathrm{O}$, thus any value hetween $32 \% \mathrm{O}$ and $60 \% \mathrm{O}$ indicates partial oxidation of material from sample A. A dramatic incruase in oxygen coritent with temperature is suggestive of an Arrhenius, behavior as expected for thermally activated processes. Figure 4 is useful for identifying temperature and time values where observable differences in oxide thickness might be detectablc in bright-field HRTEM. Results from HRTEM observations of portions of material taker from oxidation runs in figure 4 are sunmarized as follows. For temperatures less than or equal to $300^{\circ} \mathrm{C}$ no change in oxide thickness or particle structure was observed in HRTEM after 1 hour anneals. This is not surprising because oxide thickness changes predicted from measured RBS atomic \%O vaiues are within HRTEM measurcment uncertainty. At $350^{\circ} \mathrm{C}$ a slight increase in oxide thickness was measured in HRTEM, roughly corresponding to that expected from the corresponding RBS atomic oO vaiue. At $400^{\circ} \mathrm{C}$, small to medium size particles $\left(\mathrm{r}_{\mathrm{p}}<\right.$ 200 A) were completely cxidized as evidenced from observation of alumina lattice fringes from 
particle cores. One would expect oxide thicknesses to increase by about $10 \AA$ from cranges in oxygen content measured by RBS. At $450^{\circ} \mathrm{C}$, ail particles visible in bright-field HRTEM $\left(\mathrm{r}_{\mathrm{p}}<\right.$ $500 \AA$ ) were completely oxidized. Because RBS atomic $\% 0$ values were not $60 \%$ (the value corresponding to $\mathrm{Al}_{2} \mathrm{O}_{3}$ ) there must be larger particles present in the sample not observed with HRTEM that were not rompletely oxidized. The fact that sorne particles oxidize completely indicates that small particles oxidize at a faster rate than large particles. However, the pertinent question is whether the oxidation rate of small particles relative to large particles is over and above that due to surface area differences betweeiı small and large particles. Small particles may oxidize faster simply because of their large surface to volume ratios and not necessarily because of an enhanced diffusion cuefficient due to surface energy effects. In the experiment described below oxidation rate is inferred from change in mass of a sample as a function of time. Total change in sample mass is due to a combination of both oxygen adsorption and transport processes occurring at or through particle inteifaces. Effects of surface energy $\mathrm{cn}$ these processes are isolated by scaling mass changes by total surface area of different sample aliquots. Changes in surface area caused by the oxidation process have been ignored. Because UFG powder size distributions have been determineo, surface area per unit sample mass can be calculated and mass change data scaled to this value. Fur an ensemble of particles with different sizes, a measured mass change rate reflects an average of individual rates for many particles. We use differences in size distribution between samples to infer additional size effects in oxidation race of individual particles. Activation energy for oxidation is extracted from changes in atomic $\% 0$ and from mass uptake data as outlined below.

The ratio of total mass of pure Al to total mass of alumina in any given sample of Al powder is $z=\left[3(\% 0)^{-1}-5\right] / \beta$. Fractional change in mass of a sample following oxidation is ther given by $\Delta \mathrm{m} / \mathrm{m}_{0}=(\mathrm{z} 0-\mathrm{z} 1)\left[(1+\mathrm{z} 0)^{*}\left(\mathrm{Q}^{*}(1+\mathrm{zl})+\mathrm{zl}\right)\right]^{-1}$, where $(\mathrm{z} 0, \mathrm{z} 1)$ are mass ratios (before, after ) oxidation, and $Q$ equals two-thirds the ratio of aluminum to oxygen molecular weights. Thus atomic \%O data from post-oxidation RBS measurements can be converted to a form equivalent to that obtained from TGA. What is not immediately a yailable from RBS masurements, but can 
be obtained directly from TGA measurements, is the overall tume dependence of $\Delta \mathrm{m} / \mathrm{m}_{0}$. TGA measuremeits for different temperatures are shown in figure 5 for samples B and $\mathbf{C}$. These curves reveal that mass uptake generally follows a square-root time dzpendence, i.e., $\Delta \mathrm{m}_{\mathrm{m}} / \mathrm{m}_{\mathfrak{l}}=$ $\left(k_{p} \Delta t\right)^{1 / 2}$, as indicated by the dashed lines in figure $5 a, b ; k_{p}$ is the mass uptake coefficient describing a particular sample and will vary with temperarure. The deviation from a square-root time dependence observed in mass uptake for sample $B$ at $450^{\circ} \mathrm{C}$ most likely results from a change in structcre of thick oxide shells. Cracks or crystallization of the oxide layer would provide different oxidation paths that alter mass uptake rate. Having established the time dependence for the process, atomic $\% O$ data from figure 4 (sample $A$ ) are remapped to $\Delta \mathrm{m} / \mathrm{m}_{0}$ values in the manner described above and, aiong with direct endpoint $\Delta \mathrm{m}_{/} \mathrm{m}_{0}$ data for samples $B$ and $C$, are plotted in terms of $k_{p}$ in figure 6. In anticipation that $k_{p}$ follows an Arrhenius relation of the form $k_{p}=A \exp \left(-E_{a} / k T\right)$, TGA and RBS $k_{p}$ values have been plotted as $\ln \left(k_{p}\right)$ vs. 1000T-1 in figure 6. Several points can be made about the results shown in figure 6. Mass change rates obtained for the same temperature but different oxidation simes are equivalent as indicated by overlapping points in figure 6 . In all cases, mass charige coefficients obtained at a given temperature increase with decreasing average particle size, or equivalently. with increasing surface area to mass ratios. However, rate data for any given sample cannot be fit to a straight line over the whole temperature range measured. For reasons discussed previously, figure 7 is a $\ln \left(k_{\mathrm{p}}\right)$ versus $1000 \mathrm{~T}^{-1}$ plot of $\mathrm{k}_{\mathrm{p}}$ values scaled to sample surface area. The effect of scaling is to collapse the curves shown in figure 6 ontu essentially one curve which is fit well by the two dashed lines shown in figure 7. Slopes of these lines indicate a high and low temperature activation energy for oxidation of $1.7 \mathrm{eV}$ and $0.5 \mathrm{eV}$, respec:ively. However, in the low temperature region a slight variation ir activation energy is observed between different sample distributions. Below $400^{\circ} \mathrm{C}$, activation energies for samples $\mathrm{A}, \mathrm{B}$, and $\mathrm{C}$ are $0.52 \mathrm{eV}, 0.51 \mathrm{eV}$, and $0.72 \mathrm{eV}$, respectively. The following section discusses these results in comparison to mass uptake coefficients measured for flat aluminum surfaces. 


\section{Discussion}

Measurements reported here demonstrate that $U \widetilde{F G}$ particles oxidize via a square-roo: time dependence with an activation energy of $1.7 \mathrm{eV}$ for temperatures abov ; $350^{\circ} \mathrm{C}$. Previous studies have determined that the oxidation rate of nominally flat $\mathrm{Al}$ surfaces follows the same time dependence and the same activation energy as . neasured for UFG particles. ${ }^{(1-3)}$ Smeltzer ${ }^{(3)}$ has reported an activation energy of about $1.6-1.8 \mathrm{eV}$ valid from $400^{\circ} \mathrm{C}$ to $600^{\circ} \mathrm{C}$ for samples outgassed at $500^{\circ} \mathrm{C}$ and $600^{\circ} \mathrm{C}$, respectively. Activation energy for flat surfaces is observed to change in time( ${ }^{(\jmath)}$, presumably due to oxide transforming froma an amorphous to crystalline phase as the oxide layer thickens. No change in activation energy versus time has been observed in the oxidation of UFG Al powder oxidation except for very large changes in oxide thickness (sample B @ $450^{\circ} \mathrm{C}$ ). Equivalent activation energies suggests that the structure of the oxide of UFG Al particles is the same as found on flat surfaces. The picture is different at low temperatures. The break in slope in figure 7 can be understood in two different ways. One explanation is that the oxide found on as-prepared UFG Al powder is structurally different from flat surface oxide such that oxygen trausport through the UFG Al oxide layer is dramatically aifferent. At a temperature of about $350^{\circ} \mathrm{C}$ the as-prepered oxide on UFG $\mathrm{Al}$ particles then undergoes a transformation to bulk-like oxide structure. The nature of such a difference, if present, is not apparent in our HRTEM analysis. A second explanation is that there is a surface energy contribution to oxidation for sinall particles, but above $350^{\circ} \mathrm{C}$ a substantial fraction of small particles have completely oxidized. After such a point is reached, then TGA measures oxidation rate of large particles only, which are bulk-like in character. Cnder either explanation the difference in low. temperature activation energies suggests a size dependence over and above surface area considerations. Activation energy for sample $C$ was greater than the other two samples and also is the distribution with largest average particle size. On the basis of average particle size one would expect an even lower activation energy for sample $A$ than that measured. Again, such a difference may be masked due to consumption of small diameter particles capable of showing a significant mass change. Oxidation studies of monosized powder or single particles themselves 
would of course more clearly confirm the above conclusion. With regard to $\mathrm{d}_{1}=$ premise motivating this work, it is clear that oxidation ultimately would desensitize UFG AV/oxidizer mixtures, however, in practice we observe that even oxidations at $450^{\circ} \mathrm{C}$ for 6 hours (corresponding roughly to a doubling of oxide thickness) do not change ignition threshold energy significantly. Indeed, more complete ignition sensitivity studies have determined other means to desensitize UFG AV/oxidizer mixtures. Ignition sensitivity control is the subject of a separate paper. ${ }^{(6)}$

\section{Acknowledgments}

The authors wish to thank M. Nastasi (LANL, MST-4) for use of his annealing furnace, $R$. Schwarz and P. Desch (both LANL, CMS) for use and assistance with their TGA unit, and S. Murray (LANL, CST-2) for his technical assistance. RBS measurements were perfomied ai the Ion Beam Materials Laboratory of LANL. This work was supported jointly by the Department of Defense (Office of Munitions) and the Department of Energy (Defense Programs) within the Munitiuns Technology Development Program.

\section{References:}

[1] E.A. Gulbransen and W.S. Wysong, J. Phys. \& Colloid Chem., 51, 1087 (1947).

[2] A. Charlesby, Proc. Phys. Soc. London, 66, 317 (1953).

[3] W.W. Smeltzer, J. Electrochemical Soc., 103, 209 (1956).

[4] A.H. Pfund, Phys. Rev. 35, 1434 (1930).

H.C. Burger and P.H. Cittert, Z. Phys. 66, 210 (1930).

L. Harris, R.T. McGinnies, and B.M. Siegel, J. Opt. Soc. Am. 38, 582 (1948).

other references in C.G. Granqvist and R.A. Buhrman.

[5] C.G. Granqvist and R.A. Buhrman, J. Appl. Phys. 47, 2200 (1976).

R.A. Buhrman and C.G. Granqvist, J. Appl. Phys. 47, 2220 (1976). 
[6] C.E. Aumann, J.A. Martin, G.L. Skofronick, and A.S. Murray, Proceedings of the $471 \mathrm{st}$ meeting of the Insensitive Munitions Division of the American Defense Preparedness Association, Arlington, VA ADPA, 392-408 (1994).

\section{Figure Captions:}

Figure 1: HRTEM micrographs of a) an ultrafine grain Al particle revealing its protective oxide shell and of b) lattice fringes demonstrating a crystalline $\mathrm{Al}$ core.

Figure 2: HRTEM micrograph illustrating that UFG Al powder fabricated from inert-gas condensation are not monosized.

Figure 3: Particle size distribution for sample A obtained from HRTEM micrographs. Solid curve is a log-normal fit to the measured size distribution. Size distributions for samples $B$ and $C$ (not shown) are similar, but with different peak and width values.

Figure 4: Plot of atomic percent oxygen versus temperature measured with RBS for sample A following oxygen exposure for 30 minutes $(X)$ and 60 minutes $(\Delta)$.

Figure 5: Thermogravimetric analysis (TGA) curves obtained for sample B (5a) and C (5b) for different temperatures. Dashed lines are fits to the curves of the form $\Delta \mathrm{m} / \mathrm{m}_{0}=\left(\mathrm{k}_{\mathrm{p}}\right.$ $\Delta t)^{1 / 2}$. For figure clarity some fits are not shown.

Figure 6: Plot of mass uptake coefficient $k_{p}$ versus temperature on a $\ln \left(k_{p}\right)$ versus $1000 / T$ graph. Straight lines on such a curve would indicate mass uptake coefficients behave according to an Arrhenius relation.

Figure 7: Plot of $k_{p}$ values shown in figure 6 scaled to sample surface area for samples in individual TGA and RBS runs. Dashed lines are least square fits to scaled $\mathrm{k}_{\mathrm{p}}$ values in high and low temperature regions. 

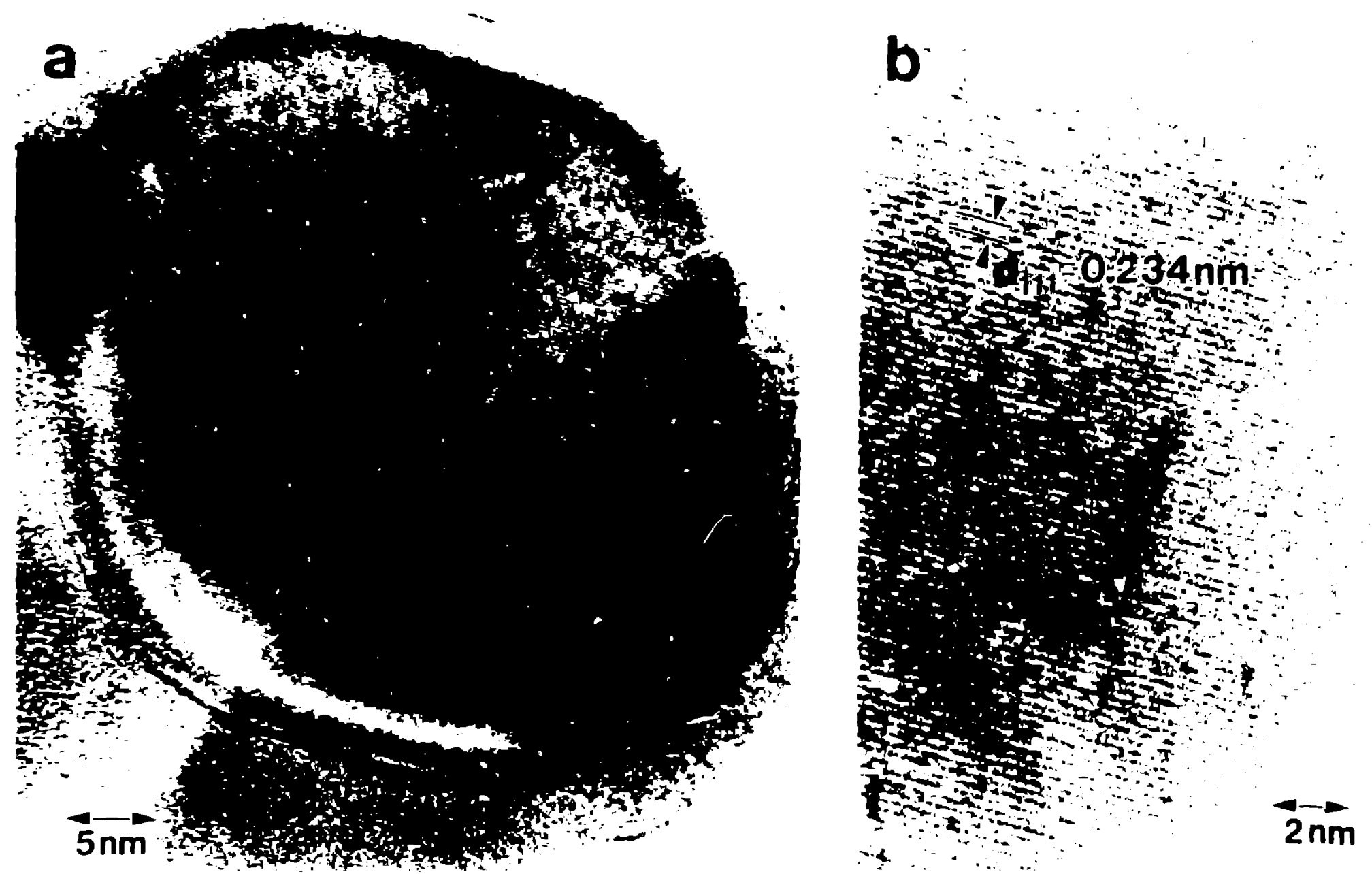


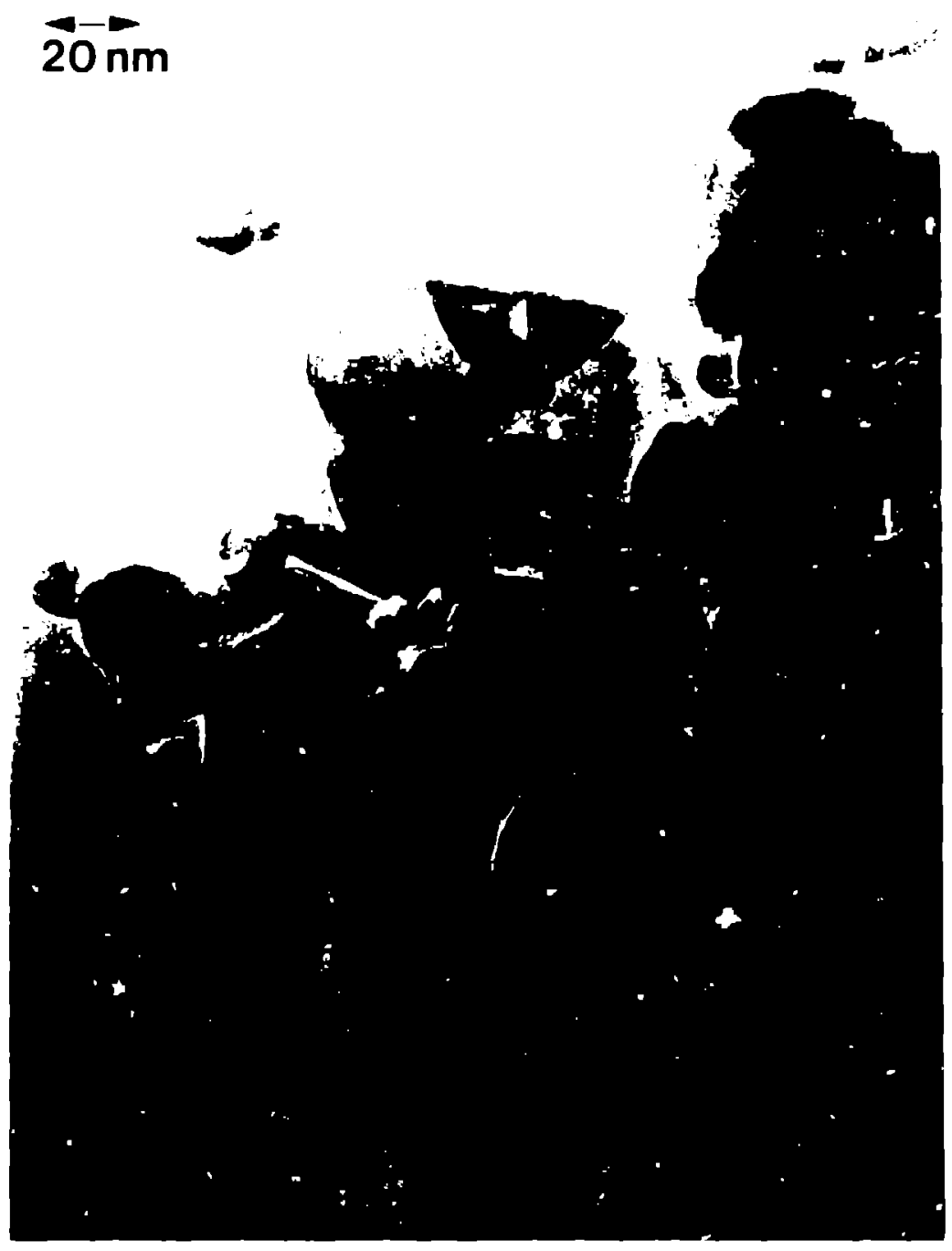




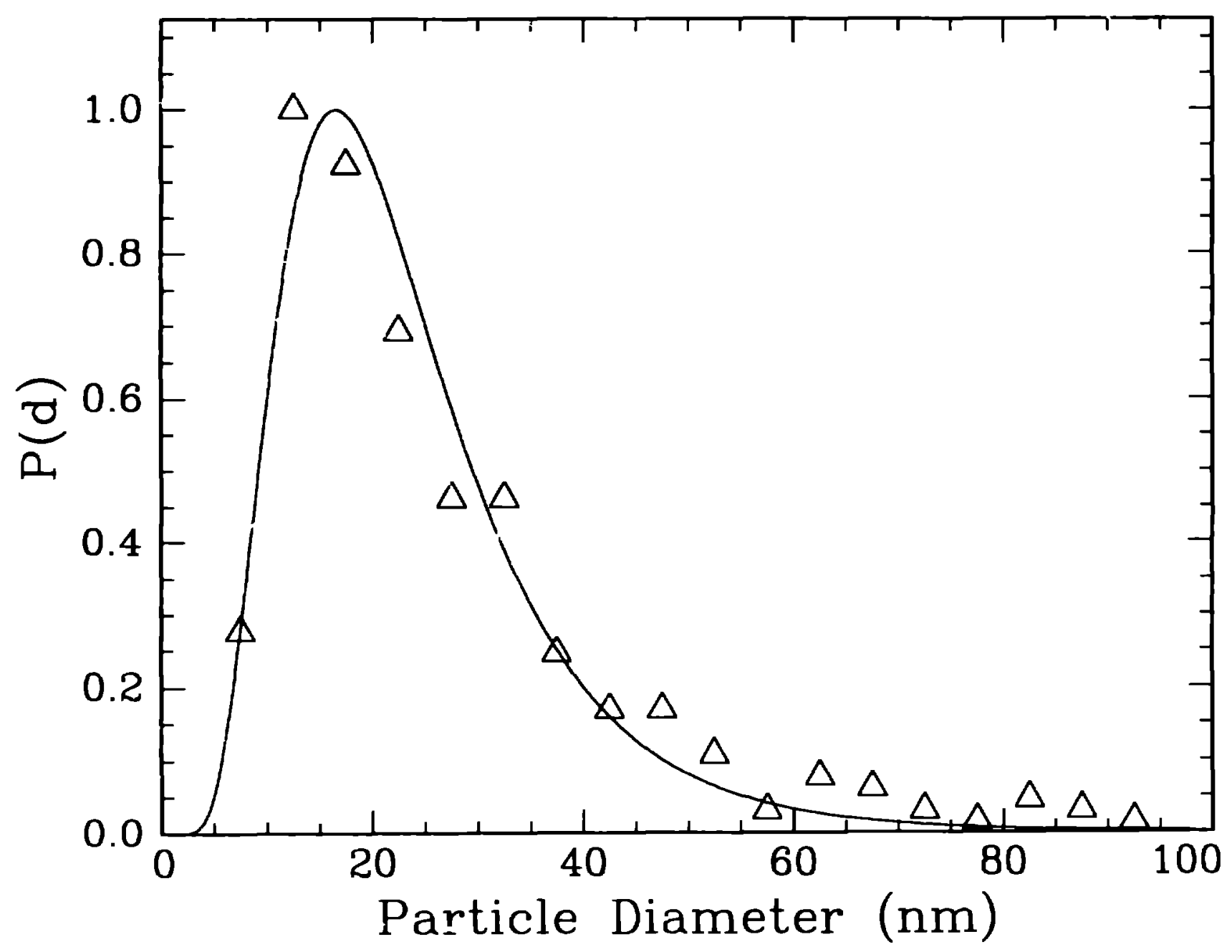




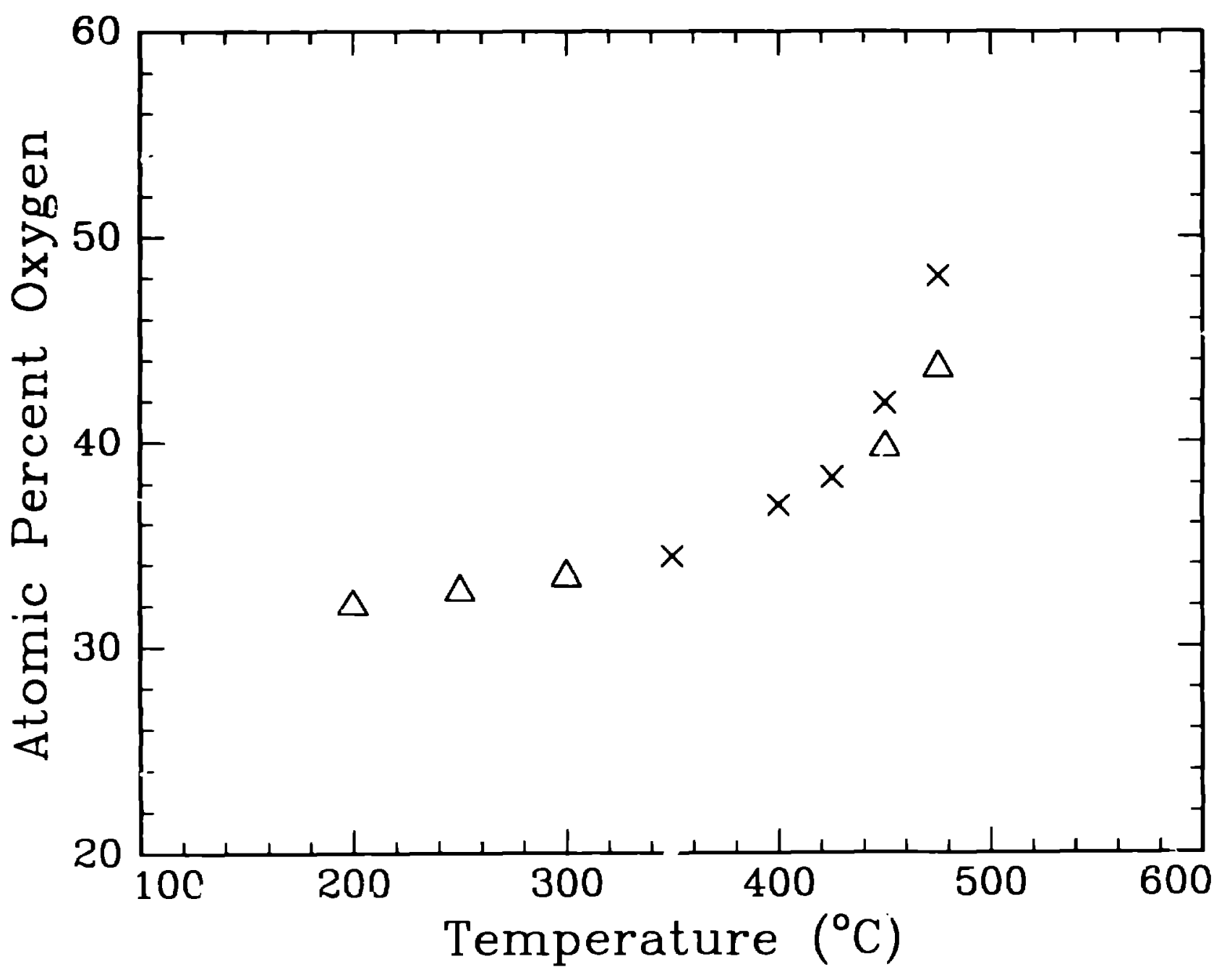



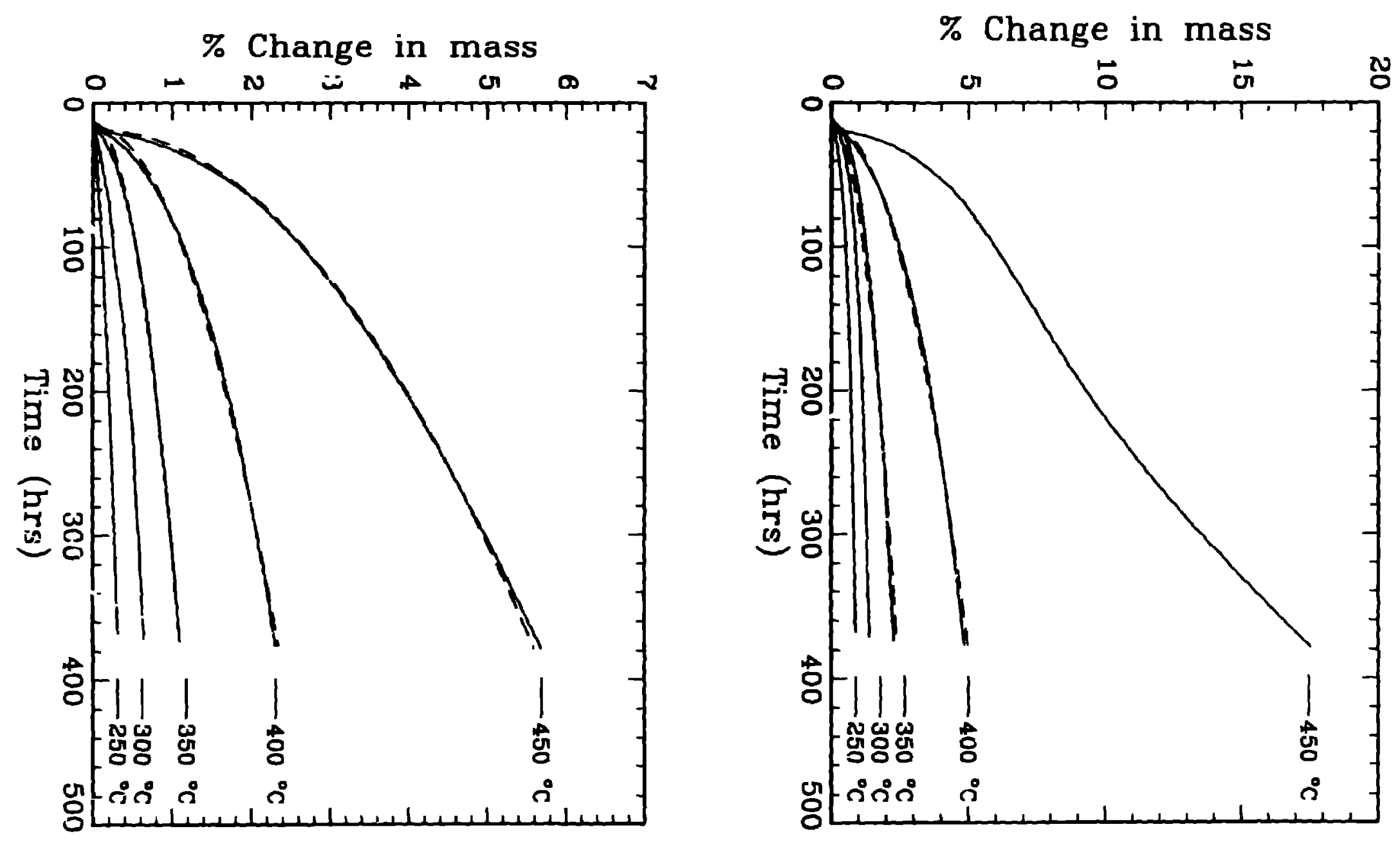


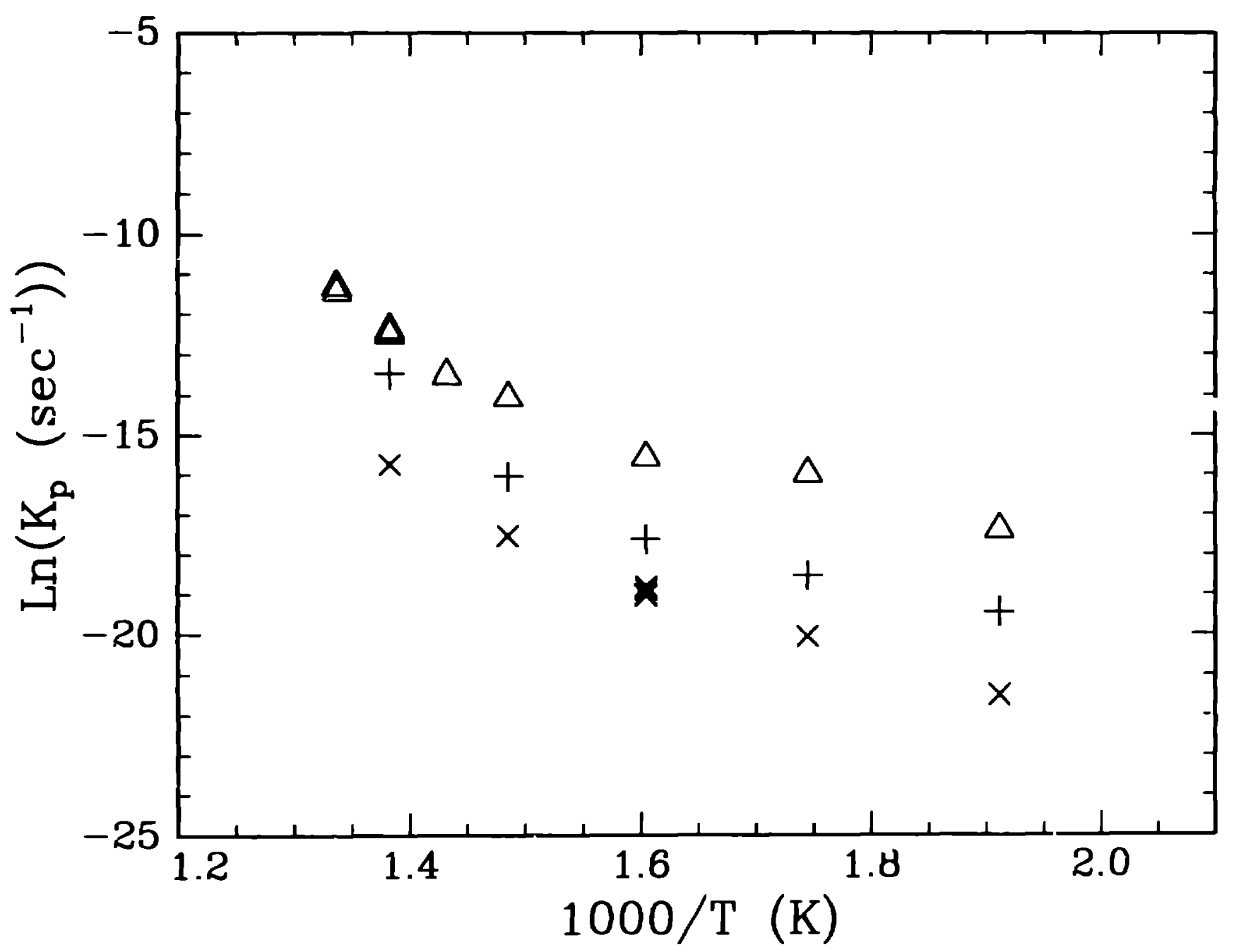




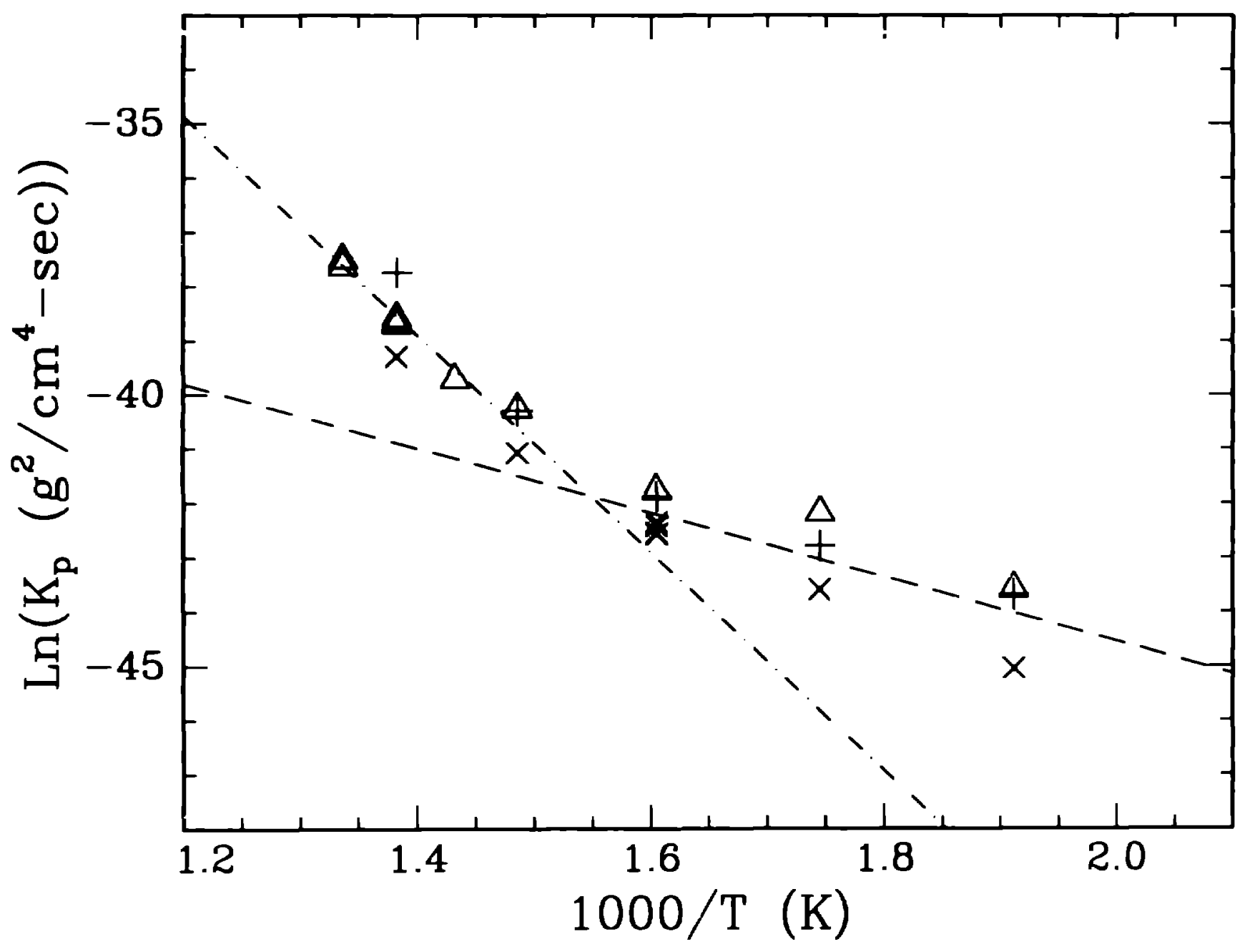

